\title{
Analysis of Load Sharing Techniques in Enterprise LTE Femtocells
}

\author{
José. M. Ruiz Avilés, S. Luna-Ramírez, M. Toril, F. Ruiz, I. de la Bandera-Cascales, P. Muñoz-Luengo \\ *University of Málaga, Communications Engineering Dept., Málaga, Spain \\ Email: \{jmruiz,sluna,mtoril,ferv,ibanderac,pabloml $\} @$ ic.uma.es
}

\begin{abstract}
In cellular networks, traffic demand is unevenly distributed both in time and space. This paper investigates the problem of re-distributing traffic demand between LTE femtocells in an enterprise scenario. A performance comparison of several traffic sharing techniques is carried out based on simulations. For this purpose, an efficient dynamic system-level LTE simulator is built. Results show that the combination of directed retry and load sharing by tuning handover margins and transmit power is an effective means to solve localized congestion problems.

Index Terms-Mobile network, traffic, load balancing, optimization, handover margins.
\end{abstract}

\section{INTRODUCTION}

In cellular networks, traffic demand is unevenly distributed both in time and space. Thus, it is common that some cells suffer from severe congestion problems, whereas their adjacent cells are underutilized. Although a proper network dimensioning avoids this problem, changes in user trends might cause that the network design become ineffective. In the long term, this problem can be solved by network re-planning strategies, such as antenna sectorization or the addition of new sites. However, in the short term, advanced Radio Resource Management (RRM) algorithms and network parameter tuning are the only means to solve this problem and make the most of the existing infrastructure.

Femtocells are a promising solution for the provision of high indoor coverage and capacity, which might help to reduce congestion problems in overloaded macrocells [1]. Femtocells are low-power base stations using cellular technology in licensed spectrum providing coverage and capacity indoors over internet-grade backhaul under operator management.

In parallel, the interest in self-organizing networks (SON) has grown in the last years with the advent of the first automatic optimization suites. A key topic in the SON literature is load balancing between cells [2][3][4]. Load balancing aims to relieve traffic congestion in the network by sharing traffic between network elements. Thus, load balancing solves congestion problems caused by localized events (e.g., football matches, shopping centres). For this purpose, the service area of cells can be modified so that the traffic demand is more evenly distributed among cells. Such an effect can be obtained by different techniques. A first group of techniques modifies

This work has been funded by the Spanish Ministry of Science and Innovation (grant TEC2009-13413) and Junta de Andalucía (grant TIC-4052). physical parameters in the base station, such as transmitted power [5] or antenna radiation pattern [6]. As these actions affect coverage, they must be handled with care. Alternatively, a second group changes parameters in RRM processes, such as Cell Re-Selection (CR) and HandOver (HO) [7]. Since tuning CR parameters is only effective during call set-up, the optimization of HO parameters, such as the HO margins, is the preferred option [2][3][4][7].

In the literature, load balancing has been studied in different scenarios (e.g., macrocell, hierarchical macro-micro) and technologies (e.g., GSM, UMTS, LTE). In [8], a load balancing scheme based on transmission power control is proposed for residential femtocells in 3GPP systems. However, to the authors' knowledge, no thorough comparison of the performance of classical load balancing techniques in femtocells has been published. Likewise, no previous work has investigated the load balancing problem in enterprise femtocells, which are expected to have several important differences with residential scenarios, namely that a) enterprise scenarios have usually a three-dimensional structure, where neighbor cells can be located everywhere around the server cell; b) a different (and probably more intense) mobility pattern; c) a higher probability of user concentration both in space (e.g., cantine) and time (e.g. coffee break, meeting end), and, d) open access instead of closed (i.e., limited) access in residential scenarios. Open access femtocells refer to those cells allowing any (potentially many) user to transmit. In this scenarios, traffic problems could arise. All these peculiarities suggest traffic sharing as a good strategy to make the most of existing femtocell network resources.

This paper evaluates the impact of different load balancing strategies on the performance of enterprise LTE femtocells. The considered schemes comprise both classical online approaches, based on RRM algorithms (e.g., directed retry, load sharing), and offline approaches, based on the network management system. Those algorithms that change cell service areas modify the transmit power and/or the handover margins of femtocells. The assessment of methods is based on a dynamic system-level LTE simulator. The main contributions of this work are: a) an efficient simulation tool for entreprise LTE femtocell scenarios, and b) a performance comparison of several classical load balancing techniques in a realistic enterprise femtocell scenario.

The rest of the paper is organized as follows. Section II 
formulates the traffic sharing problem in enterprise femtocells Section III outlines several solution techniques based on automatic network parameter tuning. Section IV describes the simulation tool used in the experiments. Section V presents the results of the performance comparison, and Section VI summarizes the main conclusions of the study.

\section{PROBlem Formulation}

In enterprise scenarios, localized traffic demand cause severe congestion problems. Even if femtocells in this scenario have similar resources, the uneven user distribution will lead to different blocking figures. Traffic sharing techniques aim to balance the traffic among different femtocells in the hope that this will decrease the overall blocking ratio, thus increasing the total carried traffic in the network.

The modification of cell service areas might reduce or increase the traffic carried by a cell. The cell service (or dominance) area is the area where any user is served by that cell. The narrowing of a cell service area decreases carried traffic, but it necessary implies the widening of neighbor cells. Changes of service area are often implemented through RRM parameter modifications. In this work, two such parameters are adjusted: handover margins and cell transmission power.

Handover margins, Margin ${ }_{P B G T}$, define by how much the signal level received from a neighbor cell must exceed that of the serving cell to trigger a handover. In the case of Power Budget (PBGT) HO, a handover is triggered when

$$
R x L E V(j)-R x L E V(i) \geq \operatorname{Margin}_{P B G T}(i, j),
$$

where $R x L E V(i)$ and $R x L E V(j)$ are the received signal level from the serving cell $i$ and neighbor cell $j$, respectively.

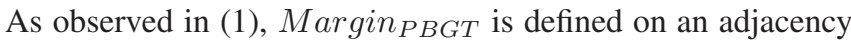
basis. Therefore, adjusting this parameter in a single adjacency only has an influence on that particular adjacency.

Cell service areas can also be modified by adjusting transmission power. A higher/lower transmission power in a base station is directly linked to higher/lower received levels in that cell. Unlike Margin $_{P B G T}$, transmission power is defined on a cell basis, so all neighbors are equally affected by changes in $i$-cell power.

In addition to the $\mathrm{HO}$ procedure, traffic sharing can also be performed by Admission Control (AC). One such schemes is Directed Retry (DR), where a blocked call in the cell where the call was initiated can be re-directed to a neighbor cell with free resources. A minimum signal level threshold, $D R_{\text {threshold, }}$ must be exceeded to be able to retry in a neighbor cell, as

$$
R x L E V(j) \geq D R_{\text {threshold }}(j) .
$$

By re-allocating users in surrounding cells, DR shares the traffic during the admission stage. Note that DR is not considered here a handover, since it occurs during the AC stage.

Regardless of the tuned parameter, an important consideration is the frequency of parameter changes. A high frequency (i.e., in the order of seconds) is desired when RRM must react to sudden changes in traffic demand, due to, e.g., start/end of a meeting in a room. In contrast, low frequencies (i.e., in the order of hours or even days) are oriented to the network reconfiguration due to some persistent unbalance between cells in the scenario (e.g., working point or new premises). Both strategies will be tested in this paper.

\section{Algorithm Outline}

In this section, different traffic sharing strategies are outlined. The main differences between them are: a) the RRM parameter to be modified (HO margins or transmit power), b) the frequency of parameter changes (slow or fast), and c) the logic behind the changes. Eight strategies are defined:

1) Benchmark - The initial scenario situation, where no parameter is adjusted and DR is disabled.

2) DR enabled - To show the influence of $D R_{\text {threshold }}$ parameter, a sensitivity analysis is performed by sweeping this parameter and the best value will be selected for subsequent methods.

3) fMLB (fast Margin Load Balance) - $\operatorname{Margin}_{P B G T}$ is modified frequently by a Fuzzy Logic Controller (FLC).

4) SMLB (slow MLB) - Similar to fMLB with slow parameter modifications.

5) fMLB+DR - Both 2) and 3) are combined; for DR, $D R_{\text {threshold }}$ is configured to a fixed value.

6) sMLB+DR - Both 2) and 4) are combined with a fixed $D R_{\text {threshold }}$ value.

7) fPLB+DR (fast Power Load Balance) - Similar to fMLB+DR, but transmission power is modified instead.

8) fPLB+fMLB+DR - Combination of three strategies, where transmit power is modified only when ping-pong $\mathrm{HO}$ is detected. For this purpose, a ping-pong parameter, $P P(i)$, is defined for cell $i$, as

$$
P P(i)=\frac{\sum_{j} N_{Q u a l H O}(j, i) \cdot\left(\operatorname{Margin}_{P B G T}(i, j)<0\right)}{\sum_{j} N_{P B G T_{-} H_{O}}(i, j)}
$$

where $N_{\text {QualHO}}(j, i)$ is the number of HOs for connection quality reasons from $j$ to $i$, and $N_{P B G T_{-} H O}(i, j)$ is the number of PBGT HOs from $i$ to $j$. $N_{Q u a l H O}(j, i)$ are only considered if handover margins are set to a negative value in such $(i, j)$ adjacency (i.e., users can be pushed to a cell $j$ with less level than cell $i$ ).

9) SPLB+sMLB+DR - Similar combination to 8) with slow parameter modifications.

\section{A. Controller for parameter tuning}

Parameter changes are implemented by an incremental FLC. As shown in Figure 1, FLC inputs are key performance indicators (e.g., cell blocking ratios) and FLC outputs are adjusted parameters (e.g., steps in HO margins). Specifically, $L R_{\text {diff } f}(i, j)$ defines the difference of load ratio between $i$ and $j$ cells and $\triangle$ Margin $_{P B G T}(i, j)(\mathrm{dB})$ is the margin modification for such adjacency $(i, j)$. An FLC consists of fuzzyfication, inference engine and defuzzyfication modules. Briefly, input indicators are labeled with some qualitative terms (e.g., 'low', 'high', 'medium') according to their numerical value and some membership functions defining the strength 


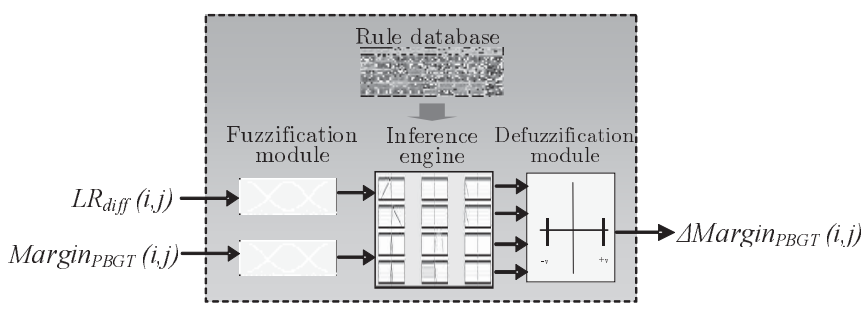

Fig. 1. Structure of fuzzy controller for tuning margins

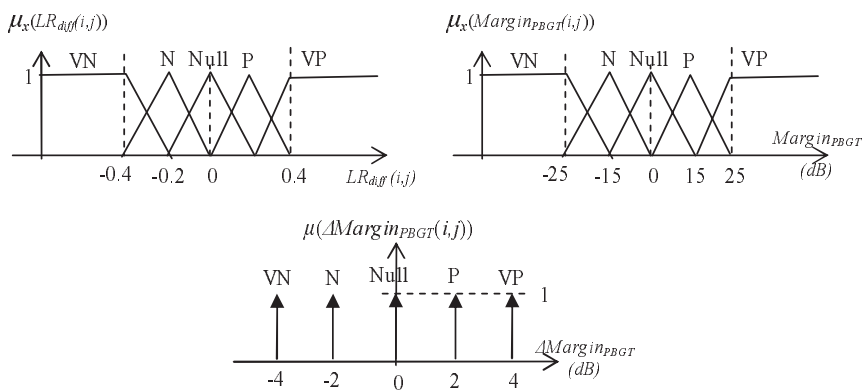

Fig. 2. Membership functions in xMLB FLCs

with which those values can be linked to the qualifier. Figure 2 presents the membership function for xMLB strategies. VN, $\mathrm{N}, \mathrm{P}$ and VP stand for Very Negative, Negative, Positive and Very Positive. The inference engine applies commonsense rules in the form of IF-THEN statements (e.g., IF load in cell 1 is 'high' AND load in cell 2 is 'low', THEN 'highly' decrease $\operatorname{Margin}_{P B G T}(1,2)$ ). Finally, the defuzzyfication module translates back the resulting qualitative action to a numerical value showing the increment or decrement of current parameter value.

FLCs in XPLB strategies use a similar FLC structure with $L R_{\text {diff }}(i, j), P P(i)$ and $P_{T X \_d i f f}(i)$ as inputs, and $\Delta P_{T X}(i)$ as output. $P_{T X_{-} \text {diff }}(i)$ defines the difference between the current and the default value of transmission power in cell $i$. As explained before, $P P(i)$ indicates the existence of ping-pong effect in cell $i$. By means of the inference machine, power is only modified when $P P(i)$ have large values. Figure 3 depicts membership functions in XPLB FLCs different from those in xMLB FLCs.

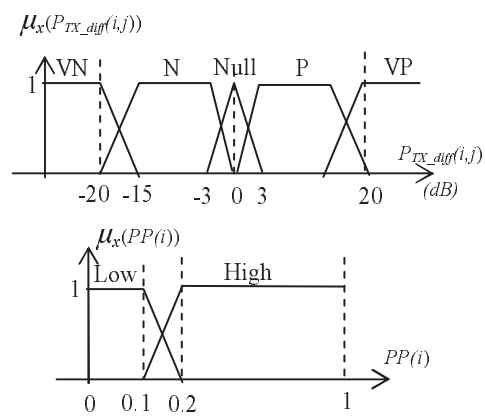

Fig. 3. Membership functions in xPLB FLCs

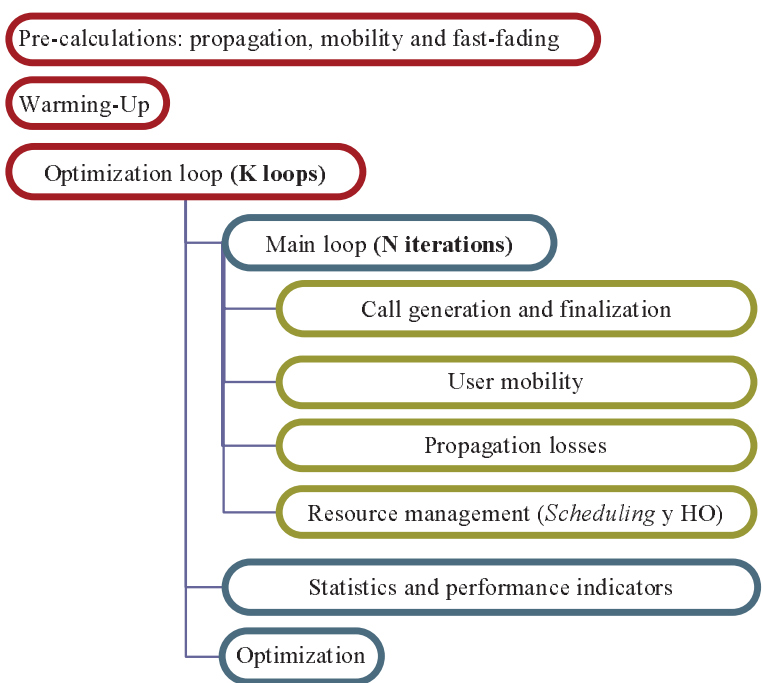

Fig. 4. Simulation software structure

\section{Simulation TOOL}

The following section describes the simulation tool with an emphasis on features either specific for femtocells or those that speed-up computations.

\section{A. Simulation structure}

Figure 4 shows the structure of the dynamic system-level simulator used in the assessment of methods. The main loop, including the most relevant tasks (i.e., mobility management, propagation/interference calculation, radio resource control) performs $\mathrm{N}$ iterations, each representing $100 \mathrm{~ms}$ of network time. After $\mathrm{N}$ iterations, network performance indicators are analyzed and a new network parameter setting is configured to improve the network behavior. By implementing an outer loop, it is possible to check changes in the network behavior after each optimization step and thus evaluate the efficacy of each optimization action. Such a tuning process is referred to as 'optimization loop'.

The radio access technology implemented is LTE. Each cell has several available Physical Resource Blocks (PRBs), where users can be scheduled in different Time SLots (TSLs). All important RRM processes are implemented (i.e., admission control, directed retry, dropped calls, packet scheduling, quality handovers, power budget handovers). The traffic source consists of voice calls with a Poisson distribution and exponential service time. Table I shows the most important properties of the simulator.

1) Propagation model: The propagation models in the simulator are based on those proposed in the WINNER II project [9]. Such models consider different environments, comprising indoor-indoor, indoor-outdoor, outdoor-indoor and outdoor-outdoor. All models share the same function structure, including important characteristics as line of sight/non line of sight, wall attenuation and diffraction, and defined by

$$
P L[d B]=A \log (d[m])+B+C \log (f[G H z] / 5)+X .
$$


TABLE I

SIMULATOR MAIN CHARACTERISTICS

\begin{tabular}{lll}
\hline $\begin{array}{l}\text { Time resolution } \\
\text { BS model }\end{array}$ & $100 \mathrm{~ms}$ & $\begin{array}{l}\text { BS femto }=13 \mathrm{dBm} \\
\text { BSmacro }=43 \mathrm{dBm}\end{array}$ \\
& Directivity & $\begin{array}{l}\text { femto: Omnidirectional } \\
\text { macro: tri-sectorial } \\
\text { macro/femto: open access }\end{array}$ \\
& Access & $9 \mathrm{~dB}$ \\
MS model & Noise figure & $-174 \mathrm{dBm} / \mathrm{Hz}$ \\
& Noise spectral density & Poisson, $0.22 \mathrm{calls} /($ user*hour) \\
Traffic model & Calls & Exponential (Av.=180 sec) \\
& Duration & Uniform \\
Mobility model & Spatial distribution & $3 \mathrm{~km} / \mathrm{h}$, random direction \& \\
& Outdoor & Rap-around \\
& Indoor & $16 \mathrm{kbps}$ \\
Service model & VOice over IP & \\
RRM model & 6 PRBs (1.4 MHZ) & \\
& Cell Reselection & \\
& Access control & PBGT, Qual, Umbrella \\
& Handover: & Time: Round-Robin (RR) \\
& Scheduler: RR-BC & Freq.: Best Channel (BC) \\
\hline
\end{tabular}

TABLE II

Propagation Model Parameters

\begin{tabular}{|c|c|c|c|c|}
\hline \multicolumn{3}{|l|}{ Scenario } & \multirow[b]{2}{*}{$\begin{array}{l}\text { Path Loss } \\
A=18.7, B=46.8, C=20 \\
A=20 B=46.4 C=20 \\
X=5 n_{w} \text { (Light wall) } \\
X=12 n_{w} \text { (Heavy wall) } \\
F L=17+4\left(n_{f}-1\right) \text { (floor att.) }\end{array}$} & \multirow{2}{*}{$\frac{\sigma_{S F}}{6}$} \\
\hline Ind2ind & A1 & $\begin{array}{l}\text { LOS } \\
\text { NLOS }\end{array}$ & & \\
\hline Ind2out & A2 & & $\begin{array}{l}P L=P L_{b}\left(d_{i n}+d_{\text {out }}\right)+ \\
P L_{t w}+P L_{i n}\left(d_{i n}\right) \\
P L_{b} \rightarrow A=22.7, B=41, C=20 \\
P L_{t w}=14+15(1-\cos (\theta)) \\
P L_{i n}=P L_{A 1}\end{array}$ & 6 \\
\hline Out2out & $\mathrm{C} 2$ & LOS & $\begin{array}{l}d<d_{B P} \rightarrow A=26 B=39 C=20 \\
d>d_{B P} \rightarrow P L=40 \log (d)+13.47+ \\
+14 \log \left(h_{B S}-1\right)-14 \log \left(h_{M S}-1\right)+ \\
+6 \log \left(f_{c} / 5\right)\end{array}$ & $\begin{array}{l}4 \\
6\end{array}$ \\
\hline & & NLOS & $\begin{array}{l}P L=\left(44-6.55 \log \left(h_{B S}\right)\right) \log (d)+ \\
+34.46+5.83 \log \left(h_{B S}\right)+23 \log \left(f_{c} / 5\right)\end{array}$ & 8 \\
\hline Out2ind & $\mathrm{C} 4$ & & $\begin{array}{l}P L=P L_{C 2}\left(d_{\text {in }}+d_{\text {out }}\right)+17.4+ \\
0.5 d_{\text {in }}-0.8 h_{M S}\end{array}$ & 8 \\
\hline
\end{tabular}

Table II shows the main parameters of the models for different environments.

2) Femtocell scenario: Figure 5 shows the simulation scenario of $3 \times 2.6 \mathrm{~km}$, comprising three tri-sectorized macrocells (blue cells) in the same site. The wrap-around technique has been implemented (black cells) to avoid border effects in the limits of the scenario. Inside the coverage area of one macrocell, an office building with dimensions $50 \mathrm{~m} \times 50 \mathrm{~m}$ (green square) has been placed. The number of floors inside the building is configurable (5 floors in this work). The floor plan is the same for all floors. Figure 6 shows the layout of one of the floors. Magenta circles reflect femtocell positions, lines are the walls (different colors represent their thickness), and black diamonds are potential terminal positions.

\section{B. Improving computational efficiency}

To speed up simulations, several calculations are performed before launching a simulation campaign.

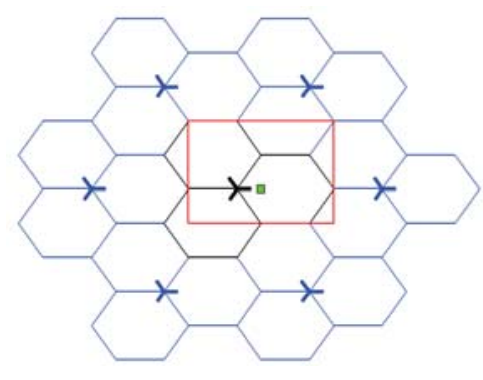

Fig. 5. Simulation scenario

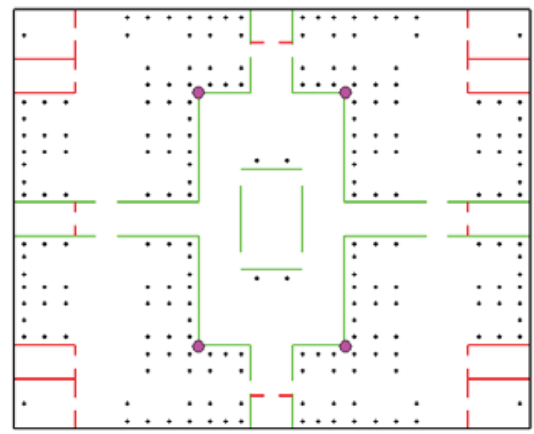

Fig. 6. A floor diagram

1) Pre-computed propagation losses: When the simulation is running, user location is updated every iteration, and therefore the power received by users from the different BSs has to be updated accordingly. Calculating this data in each iteration is a time consuming task. Alternatively, propagation losses can be computed beforehand. Such a functionality computes a matrix showing the propagation loss from each BS to a certain location. Each element of the matrix is associated with a location in the scenario, which is illustrated in Figure 7. This figure shows the attenuation in $\mathrm{dB}$ from both a macrocell and a femtocell (located at the ground floor) to every point in the scenario.

The losses values in the matrix are calculated adding three components: a) path loss (PL), b) antenna directivity, and

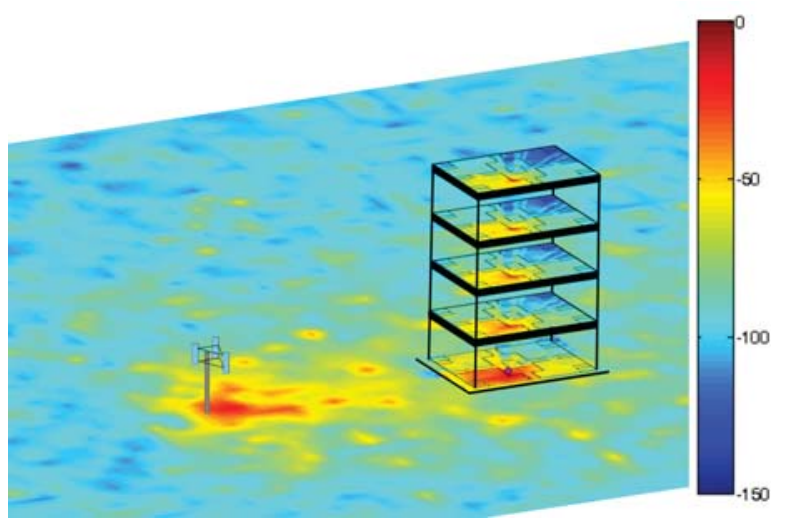

Fig. 7. Path loss attenuation grid 
TABLE III

FAST-FADING GRID PARAMETERS

\begin{tabular}{ll}
\hline Statistic distribution & Rayleigh \\
Doppler spectrum & Classic \\
Resolution & 1 wavelength $(15 \mathrm{~cm})$ \\
Grid size & $48 \mathrm{~m} \mathrm{x} 48 \mathrm{~m}(320 \lambda \times 320 \lambda)$ \\
Doppler deviation & $5.6 \mathrm{~Hz}($ vel.: $3 \mathrm{~km} / \mathrm{h})$ \\
Power profiles & EPA, EVA, ETU $(3 \mathrm{GPP}$ TS 36.101 Rel.9) \\
Bandwidths & $1.4 \mathrm{MHz}(6 \mathrm{PRBs})$ \\
Freq. samples & 4 samples per PRB \\
\hline
\end{tabular}

c) slow fading (SF). Depending on the location of the BS and the mobile station (MS), a different propagation model from those in Table II is used to calculate path loss and slow fading. For instance, the signal attenuation from a macrocell to a point inside the building is calculated by the $\mathrm{C} 4$ model. The A2 model is implemented only in a 5-meter ring around the building. For computational and memory efficiency, each model has a different spatial resolution depending on the correlation distance. In this work, the resolution is $50 \mathrm{~m}$ for $\mathrm{C} 2$ and $1 \mathrm{~m}$ for $\mathrm{A} 1, \mathrm{~A} 2$ and $\mathrm{C} 4$.

2) Pre-computed fast fading: For the same reasons given in the previous sub-section, it is necessary to pre-compute fast fading losses by constructing a grid before simulations. In this case, the grid resolution must be higher $(15 \mathrm{~cm})$ in order to capture fast fading. The large size of the scenario and the higher resolution avoids building a grid that covers the whole scenario. Instead, a grid of $48 \mathrm{~m} \mathrm{x} 48 \mathrm{~m}$ has been implemented. Such a distance is similar to the resolution of the slow fading matrix, making the reuse of the fast fading grid feasible, since the correlation of the slow fading between points at this distance is low. Table III shows the most important characteristics of the fast fading model.

3) Pre-computed indoor user mobility traces: Outdoor mobility models in simulators are often simple, since movement only needs to be reflected in a large scale. However, indoor mobility needs to be reflected more accurately, since a small movement has a strong impact on the signal levels received from base stations. A random waypoint mobility model is implemented in the simulator. In such a model, the scenario is divided into different zones. Users stay in the same position for some time, modeled as a random variable. Then, they move to a different interest point (e.g., workstation, doors... figure 6), which is selected with a certain probability. When users move, their speed is $1 \mathrm{~km} / \mathrm{h}$. Changes of floor are not considered. As in the previous sub-sections, a matrix with trajectories is precomputed and later used during the simulation.

\section{RESUlts}

\section{Simulation set-up}

The simulation scenario is an enterprise femtocell network located in a building, Figures 6 and 7. Cell traffic distribution follows a log-normal profile, i.e., cells are highly loaded at the central floor while cells at upper and lower floors are quite idle. Several congestion relief approaches are tested. Their aim is to reduce the global CBR through the load

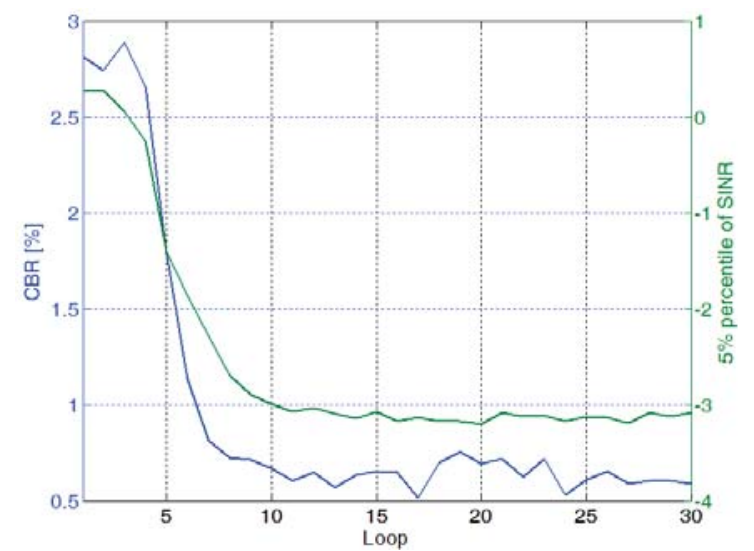

Fig. 8. Global network performance sweeping $D R_{\text {threshold }}$ value

redistribution from highly loaded cells to idle cells. To achieve this goal, the optimization techniques described in section III have been tested. About the optimization speed, there are two configurations:

Fast: 120 optimization loops of 30 seconds each. Statistics are collected over the complete simulation time, i.e., 3600 seconds. Techniques 2, 4, 6 and 9 are in this category.

Slow: 30 independent optimization loops, 1 hour of network time each. Statistics are collected after each loop, so the evolution of network performance is observed through 30 steps. Techniques 3, 5, 7 and 8 use this configuration.

A benchmark technique, without any congestion relief feature, has been first simulated for one hour by one single optimization loop. Technique 2 sweeps the parameter $D R_{\text {threshold }}$ by $3 \mathrm{~dB}$ in each loop, from $-20 \mathrm{~dB}$ to $107 \mathrm{~dB}$. For the rest of techniques, FLC processes network statistics at the end of an optimization loop and configures the new parameter value for next loop. To assess the techniques, Call Blocking Rate (CBR), Signal to Interference and Noise Ratio (SINR) and cell load are the main performance indicators.

\section{Tests Results}

The benchmark situation, first loop in Figure 8, shows a very blocked network with low connection qualities. A more detailed analysis, not shown in the figure, depicts a very unbalanced network, where cells are fully loaded (100\%) and CBR reaches a $70 \%$ figure in the central floor. At the same time, cells at lower and upper floors are quite idle ( $2 \%$ of cell load and $0 \%$ blocking ratio in some cases).

DR technique aims to find the best value for the DR minimum signal-level threshold, which is later combined with more sophisticated congestion relief algorithms. Figure 8 shows the evolution of the CBR and the $5 \%$ percentile of SINR when $D R_{\text {threshold }}$ varies from $-20 \mathrm{dBm}$ to $-107 \mathrm{dBm}$. Around $80 \mathrm{dBm}$, the indicators reach a stable situation and further increments of $D R_{\text {threshold }}$ do not produce significant changes. Hence, $-80 \mathrm{dBm}$ has been used for $D R_{\text {threshold }}$ in further simulations. 


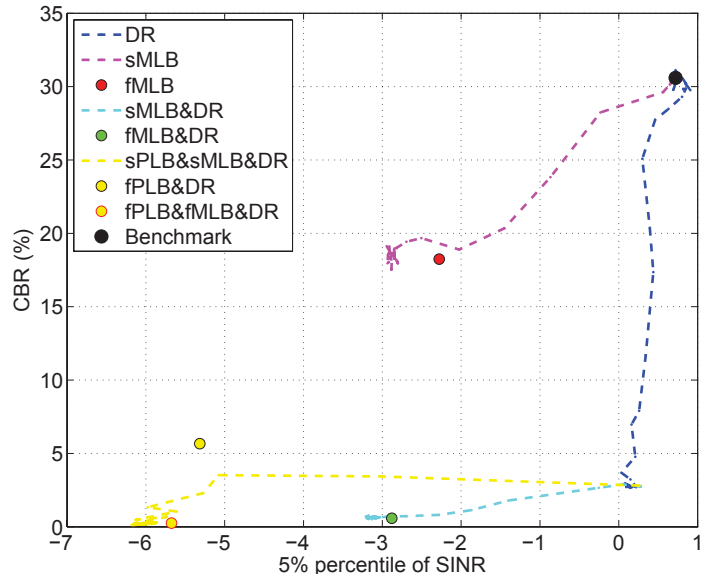

Fig. 9. Network performance comparison

Figure 9 shows the comparison for all techniques. Note that fast techniques are illustrated by one only circle (statistics are collected once for the whole simulation time), and slow techniques are drawn by a dashed line, representing network performance evolution through the optimization loops.

Generally speaking, techniques with DR algorithm reach better CBR values (lower in the figure). Techniques modifying $\mathrm{HO}$ margins (number 3, 4, 5 and 6) improve CBR figures, but, at the same time, experience worse SINR values. Terminals are redirected to farer cells so they receive very high interference from their old server cell and, consequently, lower SINR figures. High interference levels cause a lot of Quality Handovers (QualHO) going back to the best cell. This process is a clear ping-pong effect where, first, $\mathrm{HO}$ margins try to push the terminal out and, then, QualHO procedure bring it back to the original cell. This ping-pong effect prevent a strict load balance control, so load balance is not completely reached, as shown in Figure 10, where each line represents the load evolution of each cell for technique 6 .

Finally, transmission power modifications (i.e., techniques 7 to 9) try to mitigate the interference effect. This techniques decrease cell transmission power when ping-pong is being appreciated, as described in section III. However, figure 9 illustrates that these xPLB techniques experience similar BCR performance but worse SINR values.

\section{CONCLUSIONS}

The simulator presented in this paper has proven to be an efficient and effective tool. Simulation time is low enough to to test an enterprise femtocell network with several floors and an umbrella macrocell, proving the efficacy of the functions implemented to improve the simulator efficiency. By this simulator, several classical congestion relief approaches has been tested in a 3-D office scenario. These good behavior will allow the evaluation of more sophisticated optimization strategies in the future.

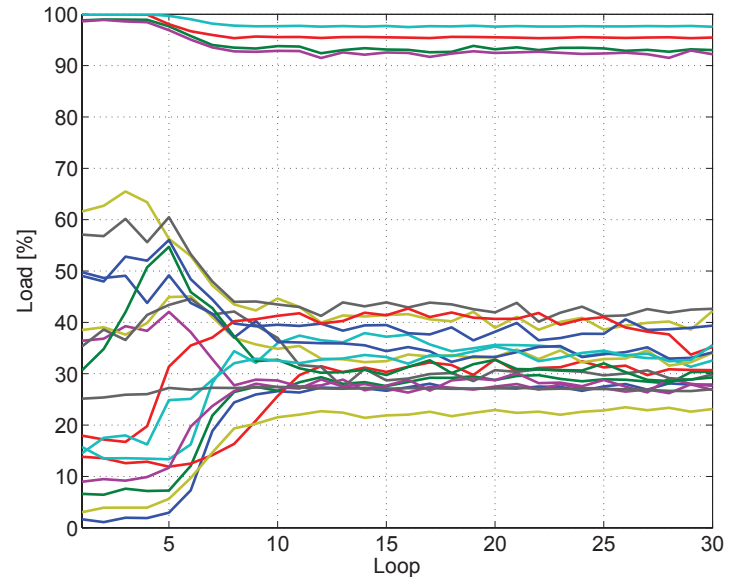

Fig. 10. Evolution of cell loads for SMLB_DR

Simulation results have shown that most congestion relief strategies reduce the CBR to some extent at the expense of deteriorating the global SINR in the network. From the results, it can be concluded that the interference between femtocells in an office scenario is an important limitation for load balancing. Unlike macrocells urban scenarios, where cell overlapping is large and changes smoothly, office scenarios show limited overlap between femtocells due to walls and, especially, the floor structure. Therefore, when users are sent to another cell to balance load, interference levels received from the original serving cell are often much larger than those received from the new serving cell. Current work aims to find more sophisticated congestion relief techniques for this challenging scenario.

\section{REFERENCES}

[1] V. Chandrasekhar, J. Andrews, and A. Gatherer, "Femtocell networks: a survey," Communications Magazine, IEEE, vol. 46, no. 9, pp. 59 -67, september 2008.

[2] A. Lobinger, S. Stefanski, T. Jansen, and I. Balan, "Load balancing in downlink lte self-optimizing networks," in Vehicular Technology Conference (VTC 2010-Spring), 2010 IEEE 71st, may 2010, pp. 1 -5.

[3] R. Kwan, R. Arnott, R. Paterson, R. Trivisonno, and M. Kubota, "On mobility load balancing for LTE systems," in Vehicular Technology Conference Fall (VTC 2010-Fall), IEEE 72nd, sept. 2010, pp. 1 -5.

[4] P. Muñoz, R. Barco, I. De la Bandera, M. Toril, and S. Luna-Ramírez, "Optimization of a fuzzy logic controller for handover-based load balancing," in Proc. of IEEE Vehicular Technology Conference (VTC), Spring 2011, 2011.

[5] J. Kojima and K. Mizoe, "Radio mobile communication system wherein probability of loss of calls is reduced without a surplus of base station equipment," U.S. Patent 4435840, Tech. Rep., 1984.

[6] D. Lee and C. Xu, "Mechanical antenna downtilt and its impact on system design," in Vehicular Technology Conference, 1997 IEEE 47th, vol. 2, may 1997, pp. $447-451$ vol.2.

[7] M. Toril and V. Wille, "Optimization of handover parameters for traffic sharing in GERAN," Wireless Personal Communications, vol. 47, no. 3, pp. 315-336, Nov 2008.

[8] K. Lee, S. Kim, S. Lee, and J. Ma, "Load balancing with transmission power control in femtocell networks," in Advanced Communication Technology (ICACT), 2011 13th International Conference on, feb. 2011, pp. $519-522$.

[9] WINNER II IST project, "D1.1.2. WINNER II channel models. part II. radio channel measurement and analysis results. v1.0," WINNER II IST project, Tech. Rep., 2007. [Online]. Available: www.ist-winner.org/ 